\title{
Population pharmacokinetics and pharmacodynamics of rivaroxaban in patients with acute coronary syndromes
}

Xu Steven $\mathrm{Xu},{ }^{1}$ Kenneth Moore, ${ }^{2}$ Paul Burton, ${ }^{3}$ Kim Stuyckens, ${ }^{4}$ Wolfgang Mueck, ${ }^{5}$ Stefaan Rossenu, ${ }^{4,+}$ Alexei Plotnikov, ${ }^{3}$

\section{Michael Gibson ${ }^{6} \&$ An Vermeulen ${ }^{4}$}

${ }^{1}$ Clinical Pharmacology, Advanced PK-PD Modeling and Simulation, Janssen Research \& Development, Titusville, NJ, USA, ${ }^{2}$ Clinical Pharmacology, Janssen Research \& Development, Titusville, NJ, USA, ${ }^{3}$ Janssen Research \& Development, Raritan, NJ, USA, ${ }^{4}$ Clinical Pharmacology, Advanced PK-PD Modeling and Simulation, Janssen Research \& Development, Beerse, Belgium, ${ }^{5}$ Bayer Pharma AG, Wuppertal, Germany and ${ }^{6}$ Cardiovascular Division, Beth Israel Hospital, Harvard Medical School, Boston, MA, USA

\section{Correspondence}

Dr Xu Steven Xu PhD, Clinical

Pharmacology, Advanced PK-PD Modeling and Simulation, Janssen Research \&

Development, Titusville, NJ 08560, USA.

Tel.: +1 9089274267

Fax:+1 9082031527

E-mail: sxu26@its.jnj.com

+Current affilliation: Ablynx nv

Zwijnaarde, Belgium

Keywords

acute coronary syndrome,

pharmacodynamics, population

pharmacokinetics, prothrombin time, rivaroxaban

\section{Received}

13 October 2011

Accepted

1 January 2012

Accepted Article

Published Online

13 January 2012

\section{WHAT IS ALREADY KNOWN ABOUT}

\section{THIS SUBJECT}

- Population pharmacokinetics and

pharmacodynamics of rivaroxaban have been

characterized in healthy subjects and in patients

with total venous thromboembolism, deep vein thrombosis or atrial fibrillation.

\section{WHAT THIS STUDY ADDS}

- This article is the first description of the population pharmacokinetics (PK) and pharmacodynamics (PD) of rivaroxaban in patients with acute coronary syndrome (ACS). It is the largest population pharmacokinetic and pharmacodynamic study on rivaroxaban conducted to date $(n=2290)$. The PK and PK-PD relationship of rivaroxaban in patients with ACS were similar to those in other patient populations. In addition, model-based simulations showed that the influence of renal function and age on the exposure to rivaroxaban in the ACS population were similar to the findings from Phase 1 special population studies. These findings suggest that rivaroxaban has highly predictable PK-PD and may provide a consistent anticoagulant effect across the studied patient populations, which allows an accurate prediction of the dose to control anticoagulation optimally.

\section{AIMS}

The aim of this analysis was to use a population approach to facilitate the understanding of the pharmacokinetics and pharmacodynamics of rivaroxaban in patients with acute coronary syndrome (ACS) and to evaluate the influence of patient covariates on the exposure of rivaroxaban in patients with ACS.

\section{METHODS}

A population pharmacokinetic model was developed using pharmacokinetic samples from 2290 patients in Anti-Xa Therapy to Lower Cardiovascular Events in Addition to Standard Therapy in Subjects with Acute Coronary Syndrome Thrombolysis in Myocardial Infarction 46. The relationship between pharmacokinetics and the primary pharmacodynamic end point, prothrombin time, was evaluated.

\section{RESULTS}

The pharmacokinetics of rivaroxaban in patients with ACS was adequately described by an oral one-compartment model. The estimated absorption rate, apparent clearance and volume of distribution were $1.24 \mathrm{~h}^{-1}$ (interindividual variability, $139 \%), 6.48 \mathrm{I} \mathrm{h}^{-1}(31 \%)$ and $57.9 \mathrm{I}(10 \%)$, respectively. Simulations indicate that the influences of renal function, age and bodyweight on exposure in ACS patients are consistent with the findings in previous Phase 1 studies. Rivaroxaban plasma concentrations exhibit a close-to-linear relationship with prothrombin time in the ACS population, with little interindividual variability. The estimated pharmacokinetic and pharmacodynamic parameters for the ACS patients were comparable to those for venous thromboembolism prevention, deep vein thrombosis and atrial fibrillation patients.

\section{CONCLUSIONS}

The similarity in pharmacokinetics/pharmacodynamics of rivaroxaban among different patient populations and the low interindividual variability in the exposure-prothrombin time relationship indicate that the anticoagulant effect of rivaroxaban is highly predictable and consistent across all the patient populations studied. 


\section{Introduction}

Rivaroxaban is an oral, direct inhibitor of Factor Xa. Rivaroxaban has previously demonstrated clinically meaningful, robust and statistically significant efficacy compared with enoxaparin for the prophylaxis of total venous thromboembolism (VTE) and major VTE in patients after elective hip replacement or elective knee replacement surgery with modestly increased rates of bleeding [1-4]. Rivaroxaban is now approved for the prophylaxis of deep vein thrombosis (DVT), which may lead to pulmonary embolism $(P E)$ in patients undergoing knee or hip replacement surgery, in the USA, the European Union and many other countries and regions. In addition, rivaroxaban is under clinical development for the treatment of stroke prevention in nonvalvular atrial fibrillation (AF) [5], prevention of VTE in hospitalized medically ill patients [6], and treatment and secondary prevention of VTE [7]. The pharmacokinetic (PK) characteristics of rivaroxaban are characterized and summarized in the approved product label [8].

Previously, structural population PK and pharmacokinetic-pharmacodynamic (PK-PD) models were developed based on data-rich information from a multiple dose-escalation study in young healthy male subjects [9]. The models were further developed and applied in three VTE prevention trials using sparse sampling and prospectively cross-validated vs. an independent cohort of patients with intensively sampled data [10,11]. Supportive evidence for the validity and robustness of the population PK and PK-PD models was provided by further analyses performed using sparse sampling data collected in patients with DVT [12] and in patients with AF [13].

Rivaroxaban is currently also under clinical development for the secondary prevention of major cardiovascular events in patients with acute coronary syndrome (ACS), a heterogeneous condition that includes ST-segment elevation myocardial infarction (STEMI), non-ST segment elevation myocardial infarction (NSTEMI) and unstable angina (UA). Inhibition of Factor Xa, because of its pivotal position in the coagulation cascade, has the potential to block the thrombotic process and, in so doing, to reduce the incidence of subsequent ACS events. In a recent study in patients with ACS Anti-Xa Therapy to Lower Cardiovascular Events in Addition to Standard Therapy in Subjects with Acute Coronary Syndrome Thrombolysis in Myocardial Infarction 46 (ATLAS ACS-TIMI 46; ClinicalTrials.gov number, NCT00402597), rivaroxaban was shown to reduce the main secondary efficacy end point of death, myocardial infarction or stroke compared with placebo [14]. The knowledge obtained from population PK and PD modelling and simulation can facilitate drug development, and may be used to optimize patient response within the therapeutic window. Although the PK and PD of rivaroxaban have been investigated in other patient populations, such knowledge and the influence of the disease and other subject demographics and pathophysiological factors on PK and PD remain unknown for patients with ACS. In addition, comparison of PK of rivaroxaban and its pharmacological effects for patients with ACS with those for other patient populations may provide information to facilitate the accurate prediction of dose and thereby provide optimal control of anticoagulation across a wide range of patient populations. The objectives of the present population analysis were as follows: (i) to describe the PK and PK-PD relationship of rivaroxaban using nonlinear mixed-effects modelling based on sparse and rich sampling data originating from the study in patients with ACS; (ii) to characterize the inter- and intra-individual variability in the PK and PK-PD model parameters of rivaroxaban in patients with ACS; (iii) to evaluate the influence of patient covariates on the PK and PK-PD of rivaroxaban in patients with ACS; and (iv) to compare the population PK and PK-PD models for rivaroxaban in the ACS patient population with those previously developed in other patient populations. Simulations were conducted to evaluate the magnitude of the covariate effects on drug exposure.

\section{Patients and methods}

\section{Study design and treatment}

The ATLAS ACS-TIMI 46 trial was a randomized, multicentre, double-blind, placebo-controlled study designed to evaluate the safety and efficacy of rivaroxaban in patients with recent ACS who received standard of care background acetyl salicylic acid (ASA) therapy without the intention to use thienopyridine therapy (Stratum 1, ASA only) or with the intention to use thienopyridine therapy (Stratum 2, ASA plus a thienopyridine). In Stratum 1, patients were randomized to the following seven treatment groups: oral rivaroxaban at $2.5,5$ or $10 \mathrm{mg}$ twice daily [5, 10 or $20 \mathrm{mg}$ total daily dose (TDD)], oral rivaroxaban at 5, 10 or $20 \mathrm{mg}$ once daily in the evening (with a placebo dose in the morning), or placebo. In Stratum 2, patients were randomized to the following nine treatment groups: oral rivaroxaban at $2.5,5,7.5$ or $10 \mathrm{mg}$ twice daily $(5,10,15$ or $20 \mathrm{mg}$ TDD), oral rivaroxaban at 5, 10, 15 or $20 \mathrm{mg}$ once daily in the evening (with a placebo dose in the morning), or placebo. The planned duration of the double-blind treatment period was 180 days. Details regarding the design of the clinical study have previously been reported [14].

\section{Blood sampling}

Sparse PK and PD samples were taken from all randomized patients predose and 1-3, 3-6 and 8-24 h after dosing on day 1 , predose on day 30 and predose and $3 \pm 1 \mathrm{~h}$ after dosing on day 180. In addition, intensive PK samples were obtained from between 4 and 19 patients enrolled into each of the rivaroxaban dose groups predose and 1, 2, 3, 4, 6,9 and $12 \mathrm{~h}$ after dosing on day 30 .

\section{Bioanalysis}

Plasma concentrations of rivaroxaban were measured by using a validated and selective liquid chromatographic 
assay coupled to tandem mass spectrometric detection with a lower limit of quantification of $0.500 \mathrm{ng} \mathrm{ml}^{-1}$. Concentration data below the lower limit of quantification were flagged in the data set and not included in the analysis. The prothrombin time (PT) was measured using freezedried rabbit brain thromboplastin (STA Neoplastin C1 Plus ${ }^{\circledR}$; Diagnostica Stago, Parsippany, NJ, USA). The absolute results are reported in seconds.

\section{Population pharmacokinetic modelling}

The structural population PK model was developed based upon a previous population PK analysis performed in patients with DVT, which was an oral one-compartment model, parameterized in terms of apparent oral clearance $(C L / F)$, apparent volume of distribution $(V / F)$, and a first-order absorption rate constant $\left(K_{\mathrm{A}}\right)$ [12]. Nonlinear mixed-effects modelling of the sparse and rich data was conducted using NONMEM ${ }^{\circledR}$ VI level 1.1 (ICON, Ellicott City, MD, USA) $[15,16]$. Log-transformed rivaroxaban plasma concentrations were fitted using the first-order conditional estimation method without interaction. Interindividual (IIV) and interoccasion (IOV) variability for pharmacokinetic parameters were evaluated using an exponential model [17]. For estimating IOV, occasions were defined by visits: day 1, day 30 and day 180 . The magnitude of residual variability in the plasma concentrations was modelled using an additive error model in the log domain. The IIV and IOV were explored for each of the PK parameters in the model during model refinement.

Predefined covariates that are biologically meaningful and have been identified from previous population analyses were included to evaluate their influence on the PK of rivaroxaban in the ACS patients. As approximately onethird of rivaroxaban is excreted unchanged by the kidneys $[18,19]$, renal function, which tends to decrease in elderly patients compared with young adults, is expected to influence the PK of rivaroxaban [20]. Both age and renal function were found to be significant factors of rivaroxaban PK in healthy subjects, in patients with DVT and $A F$, and in patients receiving rivaroxaban for VTE prevention following major orthopaedic surgeries [9-13]. Significant effects of age and bodyweight on volume of distribution have also been reported in healthy subjects and the above-mentioned patient populations [9-13]. In addition, bioavailability of rivaroxaban was demonstrated to be dose dependent in both the DVT and VTE prevention patient populations [10-12]. Therefore, the covariates included in this PK model were as follows: age and renal function [assessed via serum creatinine (SCR)] on $C L / F$, and bodyweight [expressed via lean body mass $(\mathrm{LBM})]$ and age on $V / F$. Dose dependence was estimated on relative bioavailability $(F)$ using a $2.5 \mathrm{mg}$ rivaroxaban dose as reference dose, where $F$ equals 1.

\section{Population PK/PD modelling}

The PK-PD analysis was conducted in a subgroup of patients, from whom time-matched PK and PD samples were collected. Prothrombin time was the primary PD end point in the study. Matched samples imply that the PD blood samples to determine coagulation characteristics were taken at the same times as the PK blood samples were obtained. The resulting PK-PD data set for this analysis contained 1347 patients with 6644 observations for PT matched with corresponding PK samples. A linear intercept model with a declining exponent on plasma concentration $\left(C_{\mathrm{p}}\right), \mathrm{PT}=$ Base + Slope $\times C_{\mathrm{p}}{ }^{\left(1-n \times C_{\mathrm{p}}\right)}$, was used to describe the relationship between PT and the drug exposure based on a previously developed model for DVT patients [12]. The effect of creatinine clearance was previously identified in the PK-PT model for patients with DVT [12], and was therefore included in the intercept (Base) and the exponent $(n)$ terms.

\section{Model evaluation}

The model improvement was evaluated based on goodness-of-fit criteria such as reduction in the objective function value, the agreement between the observed and predicted concentration values, and the reduction in patterns of conditional weighted residuals. To evaluate the predictive performance of the final model, population prediction corrected visual predictive checks were performed on the concentration-time data [21]. This method evaluates whether the majority (i.e. approximately $90 \%$ ) of the observed concentrations fall within the $90 \%$ simulation interval of the individual PK profiles simulated using the final PK model. Plasma concentrations of rivaroxaban in the study population were simulated 500 times using the dose and covariate data from the patients that were used in the model development data set.

\section{Monte Carlo simulation}

To evaluate the clinical relevance of the covariates on model parameters and hence on exposure, simulations of steady-state rivaroxaban exposures [area under the curve (AUC), maximal plasma concentration $\left(C_{\max }\right)$ and trough plasma concentration $\left(C_{\min }\right)$ ] were performed after multiple oral doses of $2.5 \mathrm{mg}$ twice daily (i.e. a dose regimen used in the present ACS Phase 3 study) based on the individual parameter estimates. Steady-state AUC was calculated based on the dose and estimated individual $C L / F$. Steady-state $C_{\max }$ was predicted as the maximal concentration during a dosing interval at steady state. Steady-state $C_{\min }$ was predicted as the concentration at the end of the dose interval $\left(C_{12}\right)$. The simulated exposures were compared at different covariate levels.

\section{Results}

\section{Patient characteristics and treatments}

A summary of the patient characteristics and treatments is listed in Table 1. A total of $1784(78 \%)$ male and 506 (22\%) female patients were included in the PK data set. Of 
Table 1

Demographic characteristics

\begin{tabular}{|c|c|}
\hline Characteristic & Value \\
\hline \multirow[t]{2}{*}{ Sex } & Male (78\%) \\
\hline & Female (22\%) \\
\hline \multirow[t]{4}{*}{ Race } & White (95.8\%) \\
\hline & Black (0.8\%) \\
\hline & Asian (1.7\%) \\
\hline & Others (1.7\%) \\
\hline Age (years) & $57(24-87)^{\star}$ \\
\hline Bodyweight (kg) & $84(36-181)^{*}$ \\
\hline Lean body mass $(\mathrm{kg})$ & $60.7(30.4-90.4)^{\star}$ \\
\hline Serum creatinine $\left(\mathrm{mg} \mathrm{dl}^{-1}\right)$ & $0.95(0.49-3.17)^{\star}$ \\
\hline Creatinine clearance $\left(\mathrm{ml} \mathrm{min}^{-1}\right) \dagger$ & $96.9(22.4-298)^{*}$ \\
\hline
\end{tabular}

*Values are expressed as medians (range). +Estimated with the [22] formula.

the 2290 patients in the PK analysis data set, 303 patients received a TDD of $5 \mathrm{mg}$ rivaroxaban, 1037 patients received a TDD of $10 \mathrm{mg}$ rivaroxaban, 353 patients received a TDD of $15 \mathrm{mg}$ rivaroxaban, and 597 patients received a TDD of $20 \mathrm{mg}$ rivaroxaban.

\section{Pharmacokinetic model}

The PK of rivaroxaban in patients with ACS was adequately described by an oral one-compartment model with first-order absorption and first-order elimination. Inclusion of IIV on $K_{\mathrm{A}}$ and IOV on CL/F improved the model fit $(P<0.01)$. The parameter estimates for the final pharmacokinetic model are presented in Table 2. For a typical subject, the estimated values of $C L / F, V / F$ and $K_{\mathrm{A}}$ were $6.48 \mathrm{I} \mathrm{h}^{-1}, 57.9 \mathrm{I}$ and $1.24 \mathrm{~h}^{-1}$, respectively. The IIV of $C L / F$, $V / F$ and $K_{\mathrm{A}}$ were estimated at 31,10 and $139 \%$ coefficient of variation (expressed as \%CV), respectively. The estimated IOV on CL/F was $32 \%$. An additive error model was used to describe the residual variability for the logtransformed data, and the variance was 0.35 (59\%CV). The patient covariates included in the model were age and renal function (assessed via SCR) effects on $C L / F$, and bodyweight (expressed as LBM) and age effects on $V / F$. Similar covariate effects were also observed in the DVT and AF patient populations. As age increased by 1 year from the median age of 57 years, CL/F and V/F decreased 1.1 and $0.71 \%$, respectively, whereas $C L / F$ decreased $1.5 \%$ with $0.1 \mathrm{mg} \mathrm{dl}^{-1}$ increase in SCR. There was $0.83 \%$ increase in V/F per one kilogram increase in LBM. Since creatinine clearance (CRCL) is usually used to assess renal function, and consists of information regarding both SCR and age [22], CRCL was also tested as a covariate for $C L / F$, and was found to be statistically significant. However, the model that included age and SCR separately on $C L / F$ reduced the objective function value by 48 points compared with the model with $\mathrm{CRCL}$ as the covariate. Bioavailability was found to be dependent on dose $(P<$ 0.0001 based on the Wald test), and decreased 15\% for doses $\leq 10 \mathrm{mg}$ and $29 \%$ for doses above $10 \mathrm{mg}$, relative to a $2.5 \mathrm{mg}$ dose. This significant dose dependence was confirmed by a likelihood ratio test, because the removal of dose as the covariate of bioavailability resulted in an 85 points increase in the objective function value compared with the final PK model. The estimated bioavailability relative to a $2.5 \mathrm{mg}$ dose in the patients with ACS was comparable to that estimated in the VTE prevention patients following total knee replacement.

\section{Model evaluation}

The goodness-of-fit of the final model was tested by a graphic approach. Figure 1 indicates that population and individual predicted concentrations agreed well with observed concentrations. The conditional weighted residuals are randomly scattered across the range of population predictions and time, suggesting no bias or trends in the residual error model. The prediction corrected visual predictive checks stratified for dosing regimen are displayed in Figure 2. The visual predictive check was considered adequate because the majority of the observations lie within the $90 \%$ prediction interval and the extremes of the $90 \%$ prediction interval reflect the fifth and 95 th percentiles of the observed data well. The fifth, 50th and 95th percentiles of the observations generally fall within the $90 \%$ prediction intervals of the simulated fifth, 50th and 95th percentiles. The visual predictive check plots show that the model predicted the overall concentration data in ACS treatment patients well.

\section{PK-prothrombin time (PT) model}

Rivaroxaban concentrations correlated with PT in an almost linear fashion (Figure 3). Table 3 summarizes the parameter estimates obtained from the PK-PT structural model. The baseline PT in the study population was $14 \mathrm{~s}$, and the slope of the correlation between PT and rivaroxaban plasma concentrations was $3.2 \mathrm{~s}\left(100 \mathrm{ng} \mathrm{ml}^{-1}\right)^{-1}$. There was low overall IIV for the correlation between rivaroxaban plasma concentrations and PT, with IIV in the baseline of $9.32 \%$ and in the exponent on $C_{p}$ of $6.61 \%$. The effect of renal function (expressed as $\mathrm{CRCL}$ ) on the PT model was statistically significant $(P<0.003)$, but the magnitude of its influence was small $(<9 \%$ for the $C R C L$ range in this study population). The visual predictive check plot of the PK-PT model (Figure 3) shows that the model predicted the PT-concentration data well in the ACS patients, because the majority of the observations lie within the $90 \%$ prediction interval.

\section{Discussion}

\section{Clinical relevance of $P K$ covariates}

A full covariate modelling approach was implemented in this analysis, and all the covariates were included into the base PK model at once $[23,24]$. Therefore, statistical 


\section{Table 2}

Population estimated pharmacokinetic parameters of rivaroxaban from the final pharmacokinetic model in acute coronary syndrome (ACS) treatment patients

\begin{tabular}{|c|c|c|}
\hline Parameter & Population mean (\%SE) & $\begin{array}{l}\text { Interindividual variability, } \\
\% \mathrm{CV}(\% \mathrm{SE})\end{array}$ \\
\hline$C L / F\left(I h^{-1}\right)$ & $6.48(2.21)$ & $31.3(4.72)$ \\
\hline$K_{\mathrm{A}}\left(\mathrm{h}^{-1}\right)$ & $1.24(3.28)$ & $139(0.30)$ \\
\hline$F(\text { dose } \leq 10 \mathrm{mg} \text { relative to } 2.5 \mathrm{mg})^{*}$ & $0.851(8.91)$ & - \\
\hline \multirow{2}{*}{\multicolumn{3}{|c|}{ Covariates on CL/Ft }} \\
\hline & & \\
\hline \multicolumn{3}{|l|}{ Covariates on V/F } \\
\hline Lean body mass $\left(\mathrm{kg}^{-1}\right)$ & $0.00833(13.1)$ & - \\
\hline Age $\left(\right.$ year $\left.^{-1}\right)$ & $-0.00707(16.3)$ & - \\
\hline Interoccasion variability on $C L$ & - & $32.4(5.39)$ \\
\hline Additive error & $0.352(1.09)$ & NA \\
\hline
\end{tabular}

Abbreviations: \%CV, coefficient of variation (\%); $K_{\mathrm{A}}$, first-order absorption rate constant; LBM, lean body mass; NA, not applicable; SCR, serum creatinine; \%SE, relative standard error (\%). *Bioavailability $(F)$ at $2.5 \mathrm{mg}$ equals $1 .+C L / F \times[1-0.00112 \times($ Age -57$)-0.151 \times(S C R-0.95)] . \neq V / F \times[1+0.00833 \times(L B M-60.7)-0.00707 \times($ Age -57$)]$.
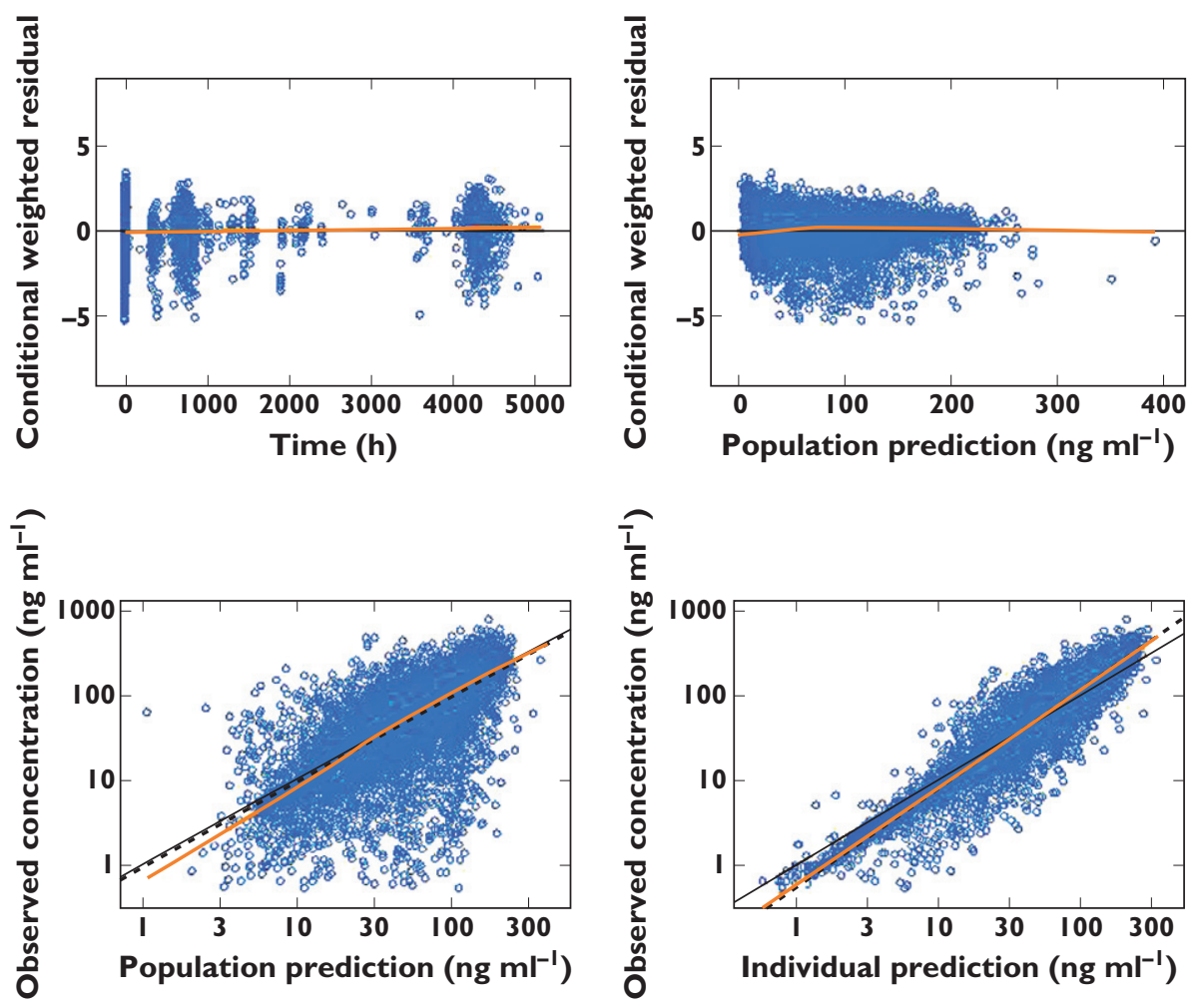

\section{Figure 1}

Diagnostic plots for the final phrmacokinetic (PK) model. The dashed line represents a locally weighted least squares. In the residual plots, the ordinate value of zero is presented (continuous horizontal line). In the plots of observed vs. population and individual predictions, the continuous line represents the line of identity

significance of the covariates was not tested based on the likelihood ratio test approach. However, statistically significant parameters/covariates can be readily identified through the Wald test, which is asymptotically equivalent to the likelihood ratio test [25]. Based on the Wald test, all the included covariates are statistically significant $(P<$ $0.0001)$, consistent with the findings from the previous population analyses in other patient populations [9-13]. 


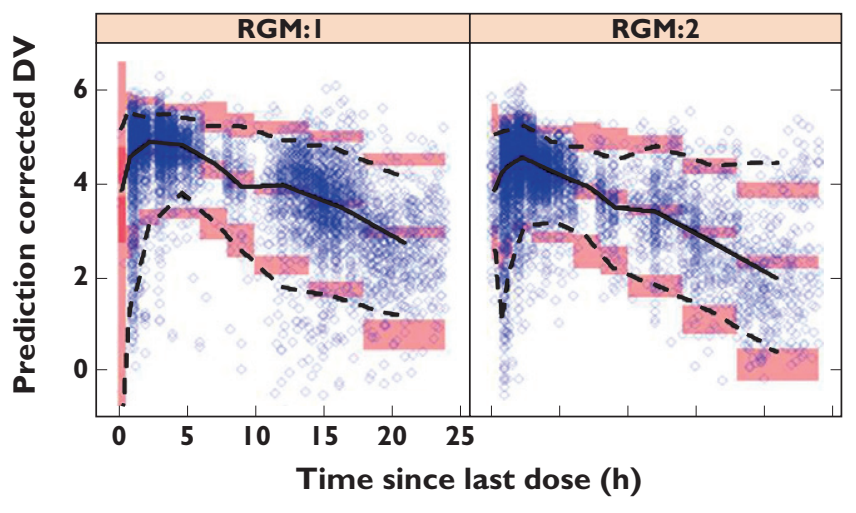

\section{Figure 2}

Visual predictive check plots for the final PK model, stratified for dosing regimen (RGM). Continuous black line is the median observed time course for rivaroxaban concentrations. The upper and lower dashed black lines represent the fifth and 95th percentiles of observed data, respectively. The semi-transparent red-shaded areas represent the simulationbased $90 \%$ confidence intervals for the fifth, 50th and 95th percentiles of the predicted data. The semi-transparent blue circles represent the observed concentrations. Abbreviations: DV, dependent variable (logtransformed concentration); RGM 1, once daily; RGM 2, twice daily

The relevance of the model covariates on drug exposures was assessed by simulations of individual steady-state drug exposures (e.g. $C_{\max } C_{\min }$ and AUC) following oral administration of a $2.5 \mathrm{mg}$ twice daily dosing regimen based on the empirical Bayes estimates of the model parameters for the ACS treatment patients in the study $(n=2290)$. Table 4 compares the median, 5 and $95 \%$ percentiles of the simulated drug exposures for subpopulations stratified for renal function (i.e. $\mathrm{CRCL}$ ), age and body mass (i.e. LBM) for the simulated $2.5 \mathrm{mg}$ twice daily dosing regimen.

Renal function was identified as a significant covariate on the PK of rivaroxaban in VTE prevention, DVT and AF populations [10-12]. Likewise, renal function was also identified as an influential covariate on the PK of rivaroxaban in the ACS population. As CRCL is commonly used to classify impairment of renal function, the comparison of exposures to rivaroxaban was made based on $\mathrm{CRCL}$, although SCR was used as the covariate for renal function in the model. The simulated median AUC in ACS treatment patients with moderate renal impairment (i.e. $30 \mathrm{ml} \mathrm{min}^{-1}$ $\leq \mathrm{CRCL}<50 \mathrm{ml} \mathrm{min}{ }^{-1}$ ) was 1.5 times that in patients with normal renal function (Table 4). This result is consistent with the findings based on the Phase 1 renal impairment study where, on average, moderately renally impaired subjects had a 1.5-fold higher AUC than subjects with normal renal function [26]. As the influence of renal function on PT was minimal $(<9 \%$ for the $C R C L$ range in this study population), the effect of $C R C L$ on the PD of rivaroxaban is mainly through its influence on the PK of rivaroxaban.

The simulated exposure to rivaroxaban increased with age. The simulated AUC in the elderly (i.e. $>75$ years old)

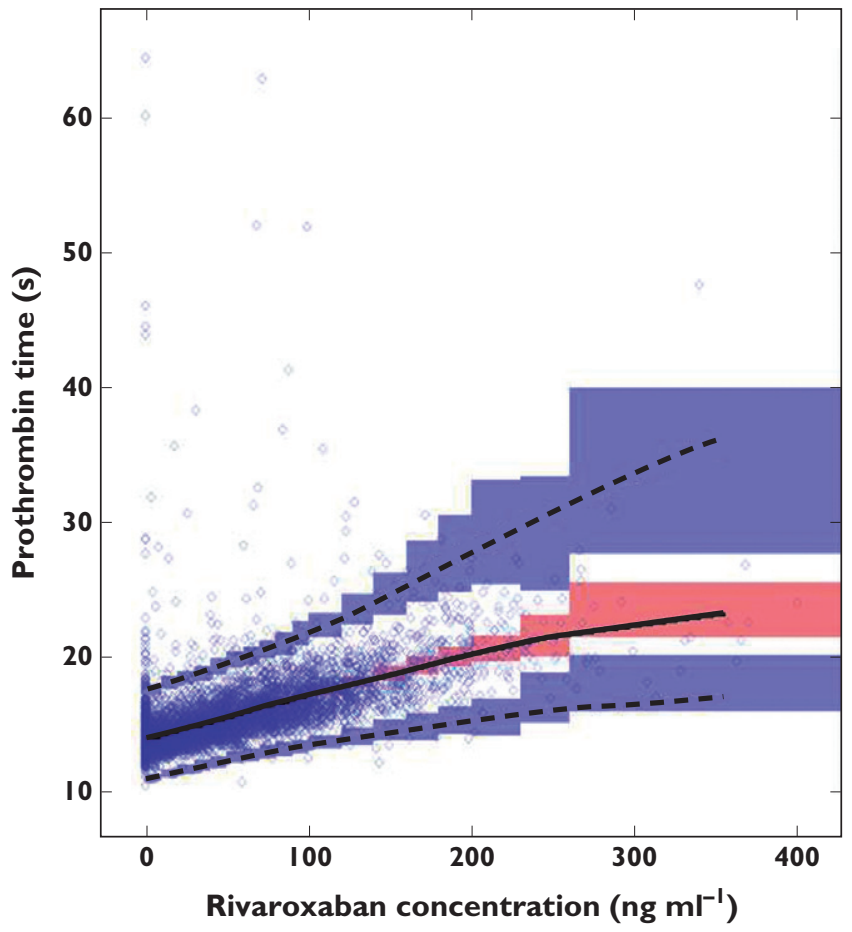

\section{Figure 3}

Visual predictive check plot for the pharmacokinetic-pharmacodynamic (PK-PD) model. Concinuous black line is the median prediction for rivaroxaban concentrations. The upper and lower dashed black lines represent the fifth and 95th percentiles of predicted data, respectively. The semitransparent red-shaded area represents the simulation-based $90 \%$ confidence intervals for the median predicted data, while the semi-transparent blue-shaded areas represent the simulation-based $90 \%$ confidence intervals for the fifth and 95th percentiles of the predicted data. The semitransparent blue circles represent the observed concentrations

was about $43 \%$ higher than that in young patients (i.e. $<50$ years old), which resembles the Phase 1 age comparison study, where the average AUC in subjects older than 75 years (up to 83 years) was $41 \%$ higher than that in young subjects (18-43 years old) $[8,27]$.The elderly-young difference can be attributed mainly to reduced renal clearance in the elderly subjects.

According to the current US Prescribing Information for patients with AF and patients for VTE prevention following major orthopaedic surgeries, rivaroxaban should be avoided if their CRCL is $<15$ and $<30 \mathrm{ml} \mathrm{min}^{-1}$, respectively [8]. Additionally, for AF patients with a CRCL level between 15 and $50 \mathrm{ml} \mathrm{min}^{-1}$, the recommended dose is reduced to $15 \mathrm{mg}$ once daily, compared with $20 \mathrm{mg}$ once daily for patients with $C R C L$ greater than $50 \mathrm{ml} \mathrm{min}^{-1}$ [8]. In the current Phase 2 study, the probability of treatmentemergent bleeding events was not statistically different between renally impaired ACS patients and the patients with normal renal function. This was confirmed by a post hoc analysis of a recent Phase 3 study (ATLAS ACS 2-TIMI 51), where no significant increase in the hazard ratio of Thrombolysis in Myocardial Infarction major bleeding 


\section{Table 3}

Final parameter estimates from population PK/PT model

\begin{tabular}{|c|c|c|}
\hline Parameter & Typical value(\%SE) & $\begin{array}{l}\text { Interindividual } \\
\text { variability (\%SE) }\end{array}$ \\
\hline Baseline (s) & $13.9(0.26)$ & $9.32(19.4)$ \\
\hline Slope $\left(\mathrm{s}\left(\mathrm{ng} \mathrm{ml}^{-1}\right)^{-1}\right)$ & $0.032(1.44)$ & NA \\
\hline Slope, describing decline of exponent on $C_{p}(n)\left[\left(\mathrm{ng} \mathrm{ml}^{-1}\right)^{-1}\right]$ & $0.0000593(23.3)$ & $6.61(17.9)$ \\
\hline Creatinine clearance on baseline $\left(\%\left(\mathrm{ml} \mathrm{min}^{-1}\right)^{-1}\right)$ & $-0.030(23.1)$ & NA \\
\hline Creatinine clearance on decline of exponent $\left.\left(\% \mathrm{ml} \mathrm{min}^{-1}\right)^{-1}\right)$ & $2.33(35.8)$ & NA \\
\hline $\mathrm{CV}$ residual error (\%SE) & & $7.6(12.1)$ \\
\hline
\end{tabular}

Abbreviations: $C_{p}$, plasma concentration; $C V$, coefficient of variation (\%); NA, not assessed; \%SE, relative standard error (\%).

\section{Table 4}

Evaluation of influence of covariates on simulated steady-state exposure for rivaroxaban following administration of a $2.5 \mathrm{mg}$ dose (twice daily) in ACS treatment patients

\begin{tabular}{|c|c|c|c|c|}
\hline Parameter & $\begin{array}{l}\text { Renal function } \\
\mathrm{CRCL}<50 \mathrm{ml} \mathrm{min}^{-1}\end{array}$ & $50 \leq \mathrm{CRCL}<80 \mathrm{ml} \mathrm{min}^{-1}$ & $\mathrm{CRCL} \geq 80 \mathrm{ml} \mathrm{min}^{-1}$ & $\begin{array}{l}\text { Ratio (CRCL }< \\
50 \mathrm{ml} \mathrm{min}^{-1} / \mathrm{CRCL} \geq \\
80 \mathrm{ml} \mathrm{min}^{-1} \text { ) }\end{array}$ \\
\hline AUC $\left(\mathrm{ng} \mathrm{h} \mathrm{ml^{-1 } )}\right.$ & $542(295-865)$ & $426(221-653)$ & 361 (209-589) & 1.50 \\
\hline$C_{\max }\left(\mathrm{ng} \mathrm{ml}^{-1}\right)$ & $63.3(38.9-90.3)$ & $51(31.1-72.1)$ & $44(27.6-66.2)$ & 1.44 \\
\hline \multirow[t]{2}{*}{$C_{\min }\left(\mathrm{ng} \mathrm{ml}^{-1}\right)$} & $27.9(8.54-53.3)$ & $19.7(6.15-38.2)$ & $16.4(5.95-33.5)$ & 1.70 \\
\hline & $\begin{array}{l}\text { Age } \\
\text { Age }<50 \text { years }\end{array}$ & $50 \leq$ age $\leq 75$ years & Age $>75$ years & $\begin{array}{l}\text { Ratio (age }>75 \\
\text { years/age }<50 \text { years) }\end{array}$ \\
\hline AUC $\left(\mathrm{ng} \mathrm{h} \mathrm{ml^{-1 } )}\right.$ & $328(183-527)$ & $397(226-654)$ & $469(277-901)$ & 1.43 \\
\hline$C_{\max }\left(\mathrm{ng} \mathrm{ml}^{-1}\right)$ & $39.9(23.9-56.9)$ & $48(30.3-72)$ & $51.3(38.9-92.9)$ & 1.29 \\
\hline \multirow[t]{2}{*}{$C_{\min }\left(\mathrm{ng} \mathrm{ml}^{-1}\right)$} & $14(5.18-30.1)$ & $18.3(6.52-38)$ & $22.6(7.7-49.9)$ & 1.61 \\
\hline & $\begin{array}{l}\text { Lean body mass } \\
\text { LBM }<53 \mathrm{~kg}\end{array}$ & $53 \leq$ LBM $<66 \mathrm{~kg}$ & LBM $\geq 66 \mathrm{~kg}$ & $\begin{array}{l}\text { Ratio }(\text { LBM < } \\
53 \mathrm{~kg} / \mathrm{LBM} \geq 66 \mathrm{~kg})\end{array}$ \\
\hline AUC (ng h ml-1) & $394(215-671)$ & $373(210-630)$ & $365(216-588)$ & 1.08 \\
\hline$C_{\max }\left(\mathrm{ng} \mathrm{ml}^{-1}\right)$ & $50.4(31.2-77.4)$ & $45.1(27.7-68)$ & $43.4(28.1-63.4)$ & 1.16 \\
\hline$C_{\min }\left(\mathrm{ng} \mathrm{ml}^{-1}\right)$ & $16.8(5.3-36.4)$ & $17(6.17-37.4)$ & $17.5(6.74-34.9)$ & 0.96 \\
\hline
\end{tabular}

Values are given as medians (5th-95th percentiles).

events (not associated with coronary artery bypass grafting) was observed in ACS patients with renal impairment, and similar rates of the primary efficacy end point were observed among the subgroups of patients with different levels of renal function [28]. Therefore, no dose adjustment according to renal function or age was warranted. However, signs or symptoms of blood loss should be observed closely and evaluated promptly in patients with moderate renal impairment (CRCL 30 to $<50 \mathrm{ml} \mathrm{min}^{-1}$ ).

The magnitude of the effect of lean body mass on the steady-state rivaroxaban exposure parameters (AUC, $C_{\max }$ and $C_{\min }$ ) was generally small (Table 4 ). The difference in AUC and $C_{\min }$ for patients with LBM $<53 \mathrm{~kg}$ (the 25th percentile of the ACS patients) and patients with LBM $\geq$ $66 \mathrm{~kg}$ (the 75th percentile) was no more than 10\%, while the $C_{\max }$ increased by $16 \%$ in patients with $\mathrm{LBM}<53 \mathrm{~kg}$ relative to patients with $L B M \geq 66 \mathrm{~kg}$ (Table 4).

\section{Comparison of parameter estimates across study populations}

Model-estimated population mean values of the PK parameters for ACS patients were generally similar to those of VTE prevention patients [10,11], DVT treatment patients [12] and patients with AF [13] (Table 5). Population mean values for $K_{\mathrm{A}}$ in patients with ACS $\left(1.24 \mathrm{~h}^{-1}\right)$ were in close agreement with those obtained in patients with $\mathrm{AF}, \mathrm{DVT}$ and the VTE prevention patients following knee and hip surgery $\left(1.16,1.23,1.20\right.$ and $1.81 \mathrm{~h}^{-1}$, respectively). It should be noted that the definition of the typical subject (i.e. median of covariate values) in each study 
Table 5

Comparison of model-based PK parameters of rivaroxaban across study populations [population mean (\% SE)]*

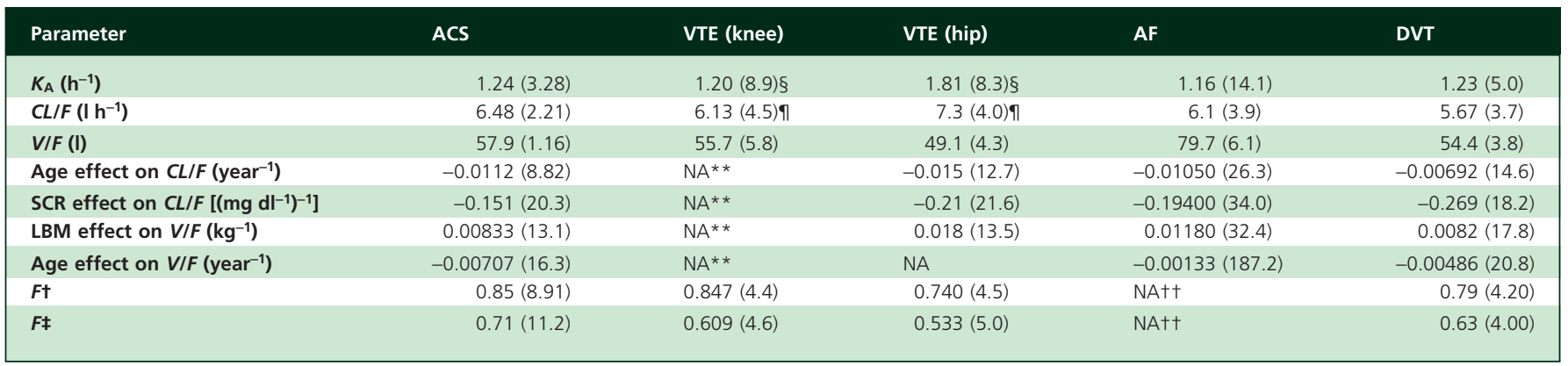

*Definitions of typical subject: ACS (age $=57$ years; $\left.S C R=0.95 \mathrm{mg} \mathrm{dl}^{-1} ; \mathrm{LBM}=61 \mathrm{~kg}\right)$; VTE knee $\left(C R C L=104 \mathrm{ml} \mathrm{min}^{-1} ; \mathrm{BSA}=1.95 \mathrm{~m}^{2}\right)$; VTE hip (age $=65$ years; SCR $=0.78 \mathrm{mg} \mathrm{dl}{ }^{-1}$; $\mathrm{LBM}=51 \mathrm{~kg}$ ); AF (age $=65$ years; SCR $=1.05 \mathrm{mg} \mathrm{dl}^{-1} ; \mathrm{LBM}=57 \mathrm{~kg}$ ); DVT (age $=61$ years; SCR $=0.94 \mathrm{mg} \mathrm{dl}^{-1} ; \mathrm{LBM}=56 \mathrm{~kg}$ ). tFor ACS, relative bioavailability of $5,7.5$ and 10 compared with $2.5 \mathrm{mg}$; for VTE (knee) and VTE (kip), relative bioavailability (F) of 5 and 10 compared with $2.5 \mathrm{mg}$; for DVT, relative bioavailability of 20 compared with $10 \mathrm{mg}$. $\neq$ For ACS, relative bioavailability of 15 and 20 compared with $2.5 \mathrm{mg}$; for VTE (knee) and VTE (hip), relative bioavailability of 20 and 30 compared with $2.5 \mathrm{mg}$; and for DVT, relative bioavailability of 30 and 40 compared with $10 \mathrm{mg}$. §Estimate for the fast absorption population based on a mixture model of $K_{\mathrm{A}}$ for the VTE (hip and knee) populations only. ICL/F estimate for study day $>3 .{ }^{*}$ Different covariates were used in this model (creatinine clearance on CL/F and body surface area on VIF). ††Only $20 \mathrm{mg}$ studied. Abbreviations: LBM, lean body mass; NA, not applicable; SCR, serum creatinine; \%SE, relative standard error (\%).

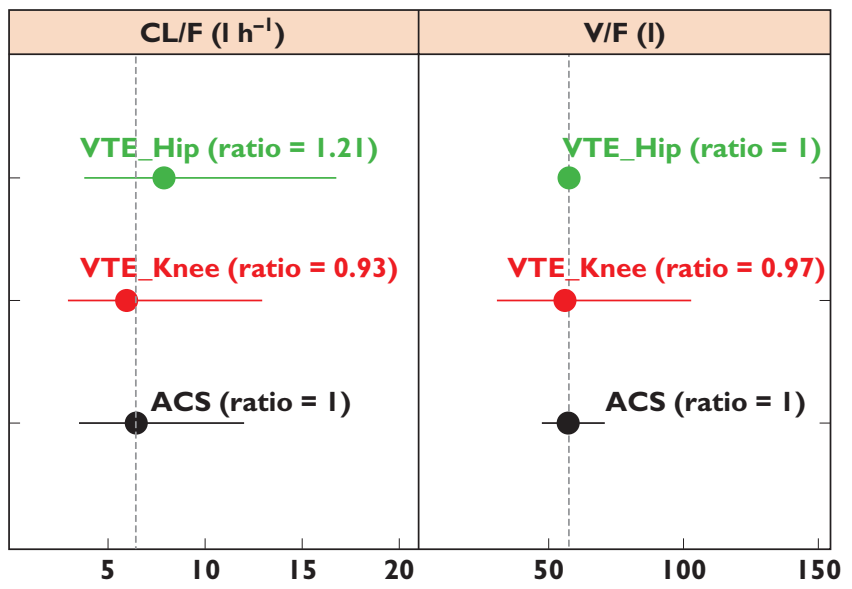

\section{Figure 4}

Comparison of estimated PK parameters for the acute coronary syndrome (ACS) population with those for a venous thromboembolism (VTE) prevention population at a $2.5 \mathrm{mg}$ dose of rivaroxaban. The confidence intervals (horizontal bars) were calculated based on interindividual variability (IIV) with a log-normal distribution assumption. Ratio of population mean values of the PK parameters were calculated using the parameter estimates for the ACS population as the reference. The IIV of apparent volume of distribution (V/F) was not included in the model in VTE prevention patients following hip surgery

population was slightly different (Table 5). Therefore, the comparison of $C L / F$ and $V / F$ across studies was done after correcting the difference in median covariate values in different patient populations (Figures 4 and 5). Figure 4 shows that the typical values of $C L / F$ and $V / F$ in patients with ACS at a dose of $2.5 \mathrm{mg}$ were comparable to those in VTE prevention patients following knee and hip surgery with a $2.5 \mathrm{mg}$ dosing regimen. The estimated $C L / F$ at $2.5 \mathrm{mg}$ in patients following total knee and hip replacement (VTE prevention) were about 7 and 20\%, respec-

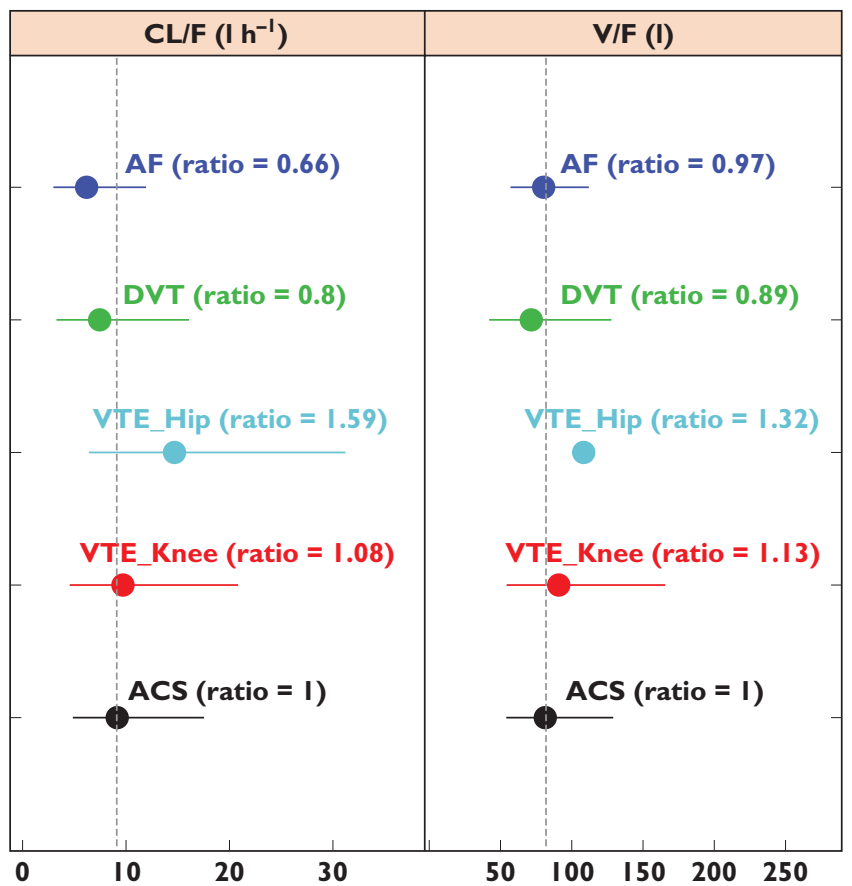

\section{Figure 5}

Comparison of estimated PK parameters across ACS, VTE prevention, deep vein thrombosis (DVT) and atrial fibrillation (AF) populations at a $20 \mathrm{mg}$ dose. The confidence intervals (horizontal bars) were calculated based on interindividual variability with a log-normal distribution assumption. Ratio of population mean values of the PK parameters were calculated using the parameter estimates for the ACS population as the reference. The IIV of V/F was not included in the model in VTE prevention patients following hip surgery

tively, lower than those estimated in the patients with ACS, whereas the estimated V/F at $2.5 \mathrm{mg}$ was virtually identical between the ACS patients and the patients after knee and hip replacement (VTE prevention; Figure 4). 


\section{Table 6}

Comparison of PT model parameter estimates across patient populations*

\begin{tabular}{|c|c|c|c|}
\hline Parameter & $\begin{array}{l}\text { AF populationt typical } \\
\text { values(\%SE) }\end{array}$ & $\begin{array}{l}\text { DVT populationt typical } \\
\text { values(\%SE) }\end{array}$ & $\begin{array}{l}\text { ACS population typical } \\
\text { values( } \% \text { SE) }\end{array}$ \\
\hline Baseline (s) & $11.4(2.0)$ & $12.5(0.7)$ & $13.9(0.26)$ \\
\hline Slope $(n)\left[\left(\mathrm{ng} \mathrm{ml}^{-1}\right)^{-1}\right]$ & $0.0000551(55.0)$ & $0.000096(7.0)$ & $0.0000593(23.3)$ \\
\hline Creatinine clearance on baseline $\left(\%\left(\mathrm{ml} \mathrm{min}^{-1}\right)^{-1}\right)$ & $0.0192(169.3)$ & $-0.04(23.2)$ & $-0.030(23.1)$ \\
\hline Creatinine clearance on $(n)\left(\%\left(m l \min ^{-1}\right)^{-1}\right)$ & $1.74(100.6)$ & $0.46(24.4)$ & $2.33(35.8)$ \\
\hline CV residual error [\%] & $12.85(18.4)$ & $10.3(8.6)$ & $7.6(12.1)$ \\
\hline
\end{tabular}

*The creatinine clearance for the typical subject in AF, DVT and ACS populations is 76,87 and $97 \mathrm{ml} \mathrm{min}^{-1}$, respectively. TThe values for AF and DVT populations are obtained from $[12,13]$. Abbreviations: CV, coefficient of variation (\%); \%SE, relative standard error (\%).

As the $2.5 \mathrm{mg}$ dose was not used in the DVT and AF populations, and a $20 \mathrm{mg}$ dose was used in the AF study, the comparison of the estimated CL/F and V/F across the ACS, AF, DVT and VTE prevention study populations was made at a $20 \mathrm{mg}$ dose of rivaroxaban (Figure 5). The $F$-values in the ACS, VTE prevention and DVT populations at the $20 \mathrm{mg}$ dose refer to those in Table 5. The differences in $V / F$ at a $20 \mathrm{mg}$ dose between patients with ACS and the other study populations were generally smaller than $15 \%$. The estimated CL/F at a $20 \mathrm{mg}$ dose for the ACS population was generally similar to that for DVT treatment and VTE prevention patients following knee surgery, because the difference was in the order of or less than $20 \%$. The estimated $C L / F$ at $20 \mathrm{mg}$ in the AF population was approximately $30 \%$ lower than that in the ACS population. Greater discrepancy was observed for the CL/F and V/F at $20 \mathrm{mg}$ between the ACS patients and the VTE prevention patients after hip surgery, where the differences were about 60 and $30 \%$, respectively. As the differences in the PK parameters were much smaller at $2.5 \mathrm{mg}$ (i.e. $~ 20 \%$ difference in $C L / F$ and virtually identical V/F; Figure 4), the larger discrepancy at $20 \mathrm{mg}$ may be a result of overcorrection of relative bioavailability, which was estimated for the combined dose strengths of 20 and $30 \mathrm{mg}$ for the VTE prevention patients undergoing hip replacement, because the relative bioavailability is expected to be lower at higher doses. Overall, the differences in population mean values across study populations are well within the interindividual variability, because the confidence intervals based on the estimated IIV for different populations largely overlapped (Figure 5).

The parameter estimates of the PK-PT model for the present ACS population are consistent with those reported for the DVT [12] and AF populations [13] (Table 6). Although the CRCL value for the typical subject in the ACS patient population was somewhat higher than those in the AF and DVT populations ( $97 \mathrm{vs.} 76$ and $87 \mathrm{ml} \mathrm{min}^{-1}$, respectively), its influence on the parameters of the typical subjects was small $(<4 \%)$. Therefore, no corrections for the difference in CRCL values for the typical subjects in these three patient populations were made. The estimated mean slope, reflecting the sensitivity of this coagulation marker towards increases in rivaroxaban drug exposure, is similar to those observed in DVT patients $\left(\left[3.6 \mathrm{~s}\left(100 \mathrm{ng} \mathrm{ml}^{-1}\right)^{-1}\right.\right.$ of rivaroxaban plasma concentration] and AF patients $\left[4.3 \mathrm{~s}\left(100 \mathrm{ng} \mathrm{m}^{-1}\right)^{-1}\right.$ of rivaroxaban plasma concentration]. The estimated mean slopes are also similar to what was observed in the VTE prevention patients $\left[3.2 \mathrm{~s}\left(100 \mathrm{ng} \mathrm{ml}^{-1}\right)^{-1}\right.$ in the hip study and $4.2 \mathrm{~s}\left(100 \mathrm{ng} \mathrm{ml}^{-1}\right)^{-1}$ in the knee study] [10, 11]. The close-tolinear relationship between rivaroxaban drug exposure and PT response also holds true for this ACS patient population, because the slope $(n)$ on the exponent was very small [0.00006 $\left.\left(\mathrm{ng} \mathrm{ml}^{-1}\right)^{-1}\right]$. Similar influences of CRCL on baseline and the slope of the exponent were observed for the ACS and the DVT populations and the ACS and the AF populations, respectively.

\section{Clinical interpretation of the findings}

Patients with a history of ACS are at higher risk for recurrent infarction, stroke and death [29]. A study found that subjects treated with the anticoagulant warfarin and ASA had a decreased rate of myocardial infarction, ischaemic stroke and revascularization compared with those treated with ASA alone [30]. However, warfarin dosing is complicated by dramatic interindividual variability in responsiveness of different patients to warfarin. While the polymorphism of CYP2C9 is associated with the highly variable metabolism of warfarin [31,32], genetic variations of the vitamin $\mathrm{K}$ epoxide reductase complex, the VKORC1 gene, have been found to be related to individual sensitivity to the anticoagulant response to warfarin therapy [33, 34]. In addition, other confounding factors, such as individual dietary habits, may further increase the inter- and/or intra-individual variability in pharmacological effects of warfarin, because genetic variants accounted for about only a third of the interindividual variability [33]. Moreover, based on the data from 48 healthy subjects, the variability in the warfarin concentrations contributed at most only $40 \%$ of the observed variability in the pharmacological anticoagulation response [35]. Bleeding events were found to be associated with the intensity of anticoagulation, and prothrombin time ratio was demonstrated to be the most predictive risk factor [36]. As a result of its large 
unexplained variability, the PK and PD (anticoagulation effect and its associated bleeding risk) of warfarin are not predictable. Therefore, the use of the same fixed dose of warfarin for all patients is not feasible. The anticoagulant effect of warfarin is usually measured by a standardized prothrombin time, and the dose is adjusted accordingly [37]. The effective daily dose of warfarin typically ranges from 0.5 to $60 \mathrm{mg}[38,39]$. Nevertheless, due to the widespread variation in the intensity of anticoagulation effect associated with warfarin, although patients taking warfarin routinely monitored their international normalized ratio readings to guide dose adjustments, they spend about a third of the treatment time outside the standard target therapeutic international normalized ratio range (2.0-3.0) [40].

Rivaroxaban exhibits only low-to-moderate interindividual variability in $V / F(\sim 10 \%)$ and $C L / F(\sim 30 \%)$ in patients with ACS. Furthermore, the prolongation in PT correlated in an almost linear fashion with plasma rivaroxaban concentration in the patient population, with little interindividual variability $(<10 \%)$. This is consistent with previous Phase 1 studies, where the observed rivaroxaban concentrations explained approximately $90 \%$ of the variability in PT prolongation $[9,41]$. Consequently, compared with warfarin, rivaroxaban is a more predictable and convenient oral anticoagulant, and can be prescribed in fixed doses without the need for routine coagulation monitoring. The similarity in the PK and PK-PD among different patient populations (i.e. VTE, DVT, AF and ACS) suggests that rivaroxaban can provide a predictable and consistent anticoagulant effect across all the patient populations studied. Therefore, the high predictability of rivaroxaban PK-PD, along with the low interindividual variation may facilitate the accurate prediction of its dose and thereby provide optimal control of anticoagulation to improve clinical outcomes and to reduce unwanted adverse events without the need to adjust the dose.

\section{Conclusion}

The PK parameter estimates for ACS patients were comparable to those for VTE prevention patients, DVT treatment patients and AF patients. The demographic covariates identified to affect rivaroxaban pharmacokinetics (CL/F and $V / F$ ) were age, renal function (assessed via serum creatinine) and bodyweight (expressed as lean body mass). The covariate estimates were consistent with those observed in VTE, DVT and AF patients. Model-based simulations showed that the influence of renal function and age on the exposure to rivaroxaban in the ACS population were similar to the findings from Phase 1 special population studies. The effect of body size (i.e. lean body mass) on the drug exposure was minimal. Prothrombin time correlated in an almost linear fashion with rivaroxaban concentrations observed in the study, with little interindividual variability. These findings suggest that rivaroxaban has highly predictable PK-PD and may provide a consistent anti- coagulant effect across the studied patient populations, which allows an accurate prediction of the dose to control anticoagulation optimally. This may improve clinical outcomes and reduce unwanted bleeding events without a need for dose adjustments.

\section{Competing Interests}

M. Gibson reports receiving research grant support from Janssen R\&D and Bayer Pharma AG, and lecture fees from Janssen R\&D. The other authors are employees of Janssen R\&D or Bayer Pharma AG. The analyses and studies described in this report were funded by Janssen R\&D and Bayer Pharma AG. Steven Xu is an adjunct assistant professor in the School of Public Health at the University of Medicine and Dentistry of New Jersey.

The authors appreciate the helpful review and constructive comments from Dr Partha Nandy.

\section{REFERENCES}

1 Lassen MR, Ageno W, Borris LC, Lieberman JR, Rosencher N, Bandel TJ, Misselwitz F, Turpie AG. Rivaroxaban versus enoxaparin for thromboprophylaxis after total knee arthroplasty. N Engl J Med 2008; 358: 2776-86.

2 Eriksson BI, Borris LC, Friedman RJ, Haas S, Huisman MV, Kakkar AK, Bandel TJ, Beckmann H, Muehlhofer E, Misselwitz F, Geerts W. Rivaroxaban versus enoxaparin for thromboprophylaxis after hip arthroplasty. N Engl J Med 2008; 358: 2765-75.

3 Kakkar AK, Brenner B, Dahl OE, Eriksson BI, Mouret P, Muntz J, Soglian AG, Pap AF, Misselwitz F, Haas S. Extended duration rivaroxaban versus short-term enoxaparin for the prevention of venous thromboembolism after total hip arthroplasty: a double-blind, randomised controlled trial. Lancet 2008; 372: 31-9.

4 Turpie AG, Lassen MR, Davidson BL, Bauer KA, Gent M, Kwong LM, Cushner FD, Lotke PA, Berkowitz SD, Bandel TJ, Benson A, Misselwitz F, Fisher WD. Rivaroxaban versus enoxaparin for thromboprophylaxis after total knee arthroplasty (RECORD4): a randomised trial. Lancet 2009; 373: 1673-80.

5 Patel MR, Mahaffey KW, Garg J, Pan G, Singer DE, Hacke W, Breithardt G, Halperin JL, Hankey GJ, Piccini JP, Becker RC, Nessel CC, Paolini JF, Berkowitz SD, Fox KA, Califf RM. Rivaroxaban versus Warfarin in Nonvalvular Atrial Fibrillation. N Engl J Med 2011; 365: 883-91.

6 Cohen A, Spiro T, Buller H, Haskell L, Hu D, Hull R, Mebazaa A, Merli G, Schellong S, Spyropoulos A, Tapson V. Rivaroxaban Compared with Enoxaparin for the Prevention of Venous Thromboembolism in Acutely III Medical Patients. New Orleans, LA: American College of Cardiology, 2011. 
7 Bauersachs R, Berkowitz SD, Brenner B, Buller HR, Decousus $H$, Gallus AS, Lensing AW, Misselwitz F, Prins $M H$, Raskob GE, Segers A, Verhamme P, Wells P, Agnelli G, Bounameaux H, Cohen A, Davidson BL, Piovella F, Schellong S. Oral rivaroxaban for symptomatic venous thromboembolism. N Engl J Med 2010; 363: 2499-510.

8 Xarelto (R) Product Label. 2011. Available at: http://www. xareltohcp.com/sites/default/files/pdf/xarelto_0. pdf\#zoom=100 (last accessed 9 May 2011).

9 Mueck W, Becka M, Kubitza D, Voith B, Zuehlsdorf M. Population model of the pharmacokinetics and pharmacodynamics of rivaroxaban - an oral, direct factor xa inhibitor - in healthy subjects. Int J Clin Pharmacol Ther 2007; 45: 335-44.

10 Mueck W, Borris LC, Dahl OE, Haas S, Huisman MV, Kakkar AK, Kalebo P, Muelhofer E, Misselwitz F, Eriksson BI. Population pharmacokinetics and pharmacodynamics of once- and twice-daily rivaroxaban for the prevention of venous thromboembolism in patients undergoing total hip replacement. Thromb Haemost 2008; 100: 453-61.

11 Mueck W, Eriksson BI, Bauer KA, Borris L, Dahl OE, Fisher WD, Gent M, Haas S, Huisman MV, Kakkar AK, Kalebo P, Kwong LM, Misselwitz F, Turpie AG. Population pharmacokinetics and pharmacodynamics of rivaroxaban an oral, direct factor Xa inhibitor - in patients undergoing major orthopaedic surgery. Clin Pharmacokinet 2008; 47: 203-16.

12 Mueck W, Lensing AW, Agnelli G, Decousus H, Prandoni P, Misselwitz F. Rivaroxaban: population pharmacokinetic analyses in patients treated for acute deep vein thrombosis and exposure simulations in patients with atrial fibrillation treated for stroke prevention. Clin Pharmacokinet 2011; 50: 675-86.

13 Girgis IG, Peters G, Moore K, Patel MR, Mahaffey KW, Nessel CC, Paolini JF, Halperin JL, Califf RM, Fox KAA, Becker RC. Population pharmacokinetics and pharmacodynamics of rivaroxaban in patients with non-valvular atrial fibrillation. American Heart Association Annual Meeting; 2011 Nov 12-16, 2011; Orlando, Fla.; 2011.

14 Mega JL, Braunwald E, Mohanavelu S, Burton P, Poulter R, Misselwitz F, Hricak V, Barnathan ES, Bordes P, Witkowski A, Markov V, Oppenheimer L, Gibson CM. Rivaroxaban versus placebo in patients with acute coronary syndromes (ATLAS ACS-TIMI 46): a randomised, double-blind, phase II trial. Lancet 2009; 374: 29-38.

15 Boeckman A, Sheiner LB, Beal SL. NONMEM VI. Ellicott City, MD: GloboMax, Icon Development Solutions, 2007.

16 Beal SL, Sheiner LB. NONMEM Users Guides. Ellicott City, MD: GloboMax, Icon Development Solutions, 1989; 1998.

17 Karlsson MO, Sheiner LB. The importance of modeling interoccasion variability in population pharmacokinetic analyses. J Pharmacokinet Biopharm 1993; 21: 735-50.

18 Weinz C, Schwarz T, Kubitza D, Mueck W, Lang D. Metabolism and excretion of rivaroxaban, an oral, direct factor $\mathrm{Xa}$ inhibitor, in rats, dogs, and humans. Drug Metab Dispos 2009; 37: 1056-64.
19 Lang D, Freudenberger C, Weinz C. In vitro metabolism of rivaroxaban, an oral, direct factor Xa inhibitor, in liver microsomes and hepatocytes of rats, dogs, and humans. Drug Metab Dispos 2009; 37: 1046-55.

20 Clark B. Biology of renal aging in humans. Adv Ren Replace Ther 2000; 7: 11-21.

21 Bergstrand M, Hooker AC, Wallin JE, Karlsson MO. Prediction-corrected visual predictive checks for diagnosing nonlinear mixed-effects models. AAPS J 2011; 13: 143-51.

22 Cockcroft DW, Gault MH. Prediction of creatinine clearance from serum creatinine. Nephron 1976; 16: 31-41.

23 Agoram B, Heatherington AC, Gastonguay MR. Development and evaluation of a population pharmacokineticpharmacodynamic model of darbepoetin alfa in patients with nonmyeloid malignancies undergoing multicycle chemotherapy. AAPS J 2006; 8: E552-63.

$24 \mathrm{Hu} \mathrm{C}$, Zhou H. An improved approach for confirmatory phase III population pharmacokinetic analysis. J Clin Pharmacol 2008; 48: 812-22.

25 Engle RF. Wald, likelihood ratio, and lagrange multiplier tests in econometrics. In: Handbook of Econometrics: Elsiever, eds Intriligator MD, Griliches Z. North-Holland Publishing Company: Amsterdam, The Netherlands, 1983; 796-801.

26 Kubitza D, Becka M, Mueck W, Halabi A, Maatouk H, Klause N, Lufft V, Wand DD, Philipp T, Bruck H. Effects of renal impairment on the pharmacokinetics, pharmacodynamics and safety of rivaroxaban, an oral, direct Factor Xa inhibitor. Br J Clin Pharmacol 2010; 70: 703-12.

27 Kubitza D, Becka M, Roth A, Mueck W. Dose-escalation study of the pharmacokinetics and pharmacodynamics of rivaroxaban in healthy elderly subjects. Curr Med Res Opin 2008; 24: 2757-65.

28 Mega JL, Braunwald E, Wiviott SD, Bassand JP, Bhatt DL, Bode C, Burton P, Cohen M, Cook-Bruns N, Fox KA, Goto S, Murphy SA, Plotnikov AN, Schneider D, Sun X, Verheugt FW, Gibson CM. Rivaroxaban in Patients with a Recent Acute Coronary Syndrome. N Engl J Med 2012; 366: 9-19.

29 Moss AJ, Benhorin J. Prognosis and management after a first myocardial infarction. N Engl J Med 1990; 322: 743-53.

30 Rothberg MB, Celestin C, Fiore LD, Lawler E, Cook JR. Warfarin plus aspirin after myocardial infarction or the acute coronary syndrome: meta-analysis with estimates of risk and benefit. Ann Intern Med 2005; 143: 241-50.

31 Takahashi H, Kashima T, Nomizo Y, Muramoto N, Shimizu T, Nasu K, Kubota T, Kimura S, Echizen H. Metabolism of warfarin enantiomers in Japanese patients with heart disease having different CYP2C9 and CYP2C19 genotypes. Clin Pharmacol Ther 1998; 63: 519-28.

32 Aithal GP, Day CP, Kesteven PJ, Daly AK. Association of polymorphisms in the cytochrome P450 CYP2C9 with warfarin dose requirement and risk of bleeding complications. Lancet 1999; 353: 717-9.

33 D'Andrea G, D'Ambrosio RL, Di Perna P, Chetta M, Santacroce R, Brancaccio V, Grandone E, Margaglione M. A 
polymorphism in the VKORC1 gene is associated with an interindividual variability in the dose-anticoagulant effect of warfarin. Blood 2005; 105: 645-9.

34 Rost S, Fregin A, Ivaskevicius V, Conzelmann E, Hortnagel K, Pelz HJ, Lappegard K, Seifried E, Scharrer I, Tuddenham EG, Muller CR, Strom TM, Oldenburg J. Mutations in VKORC1 cause warfarin resistance and multiple coagulation factor deficiency type 2. Nature 2004; 427: 537-41.

35 Pitsiua M, Parkera EM, Aarons L, Rowland M. Population pharmacokinetics and pharmacodynamics of warfarin in healthy young adults. Eur J Pharm Sci 1993; 1: 151-7.

36 Fihn SD, Callahan CM, Martin DC, McDonell MB, Henikoff JG, White $\mathrm{RH}$. The risk for and severity of bleeding complications in elderly patients treated with warfarin. The National Consortium of Anticoagulation Clinics. Ann Intern Med 1996; 124: 970-9.

37 Hirsh J, Dalen JE, Deykin D, Poller L, Bussey H. Oral anticoagulants. Mechanism of action, clinical effectiveness, and optimal therapeutic range. Chest 1995; 108: (4 Suppl.): 231S-46S.
38 James AH, Britt RP, Raskino CL, Thompson SG. Factors affecting the maintenance dose of warfarin. J Clin Pathol 1992; 45: 704-6.

39 Hallak HO, Wedlund PJ, Modi MW, Patel IH, Lewis GL, Woodruff $B$, Trowbridge AA. High clearance of (S)-warfarin in a warfarin-resistant subject. Br J Clin Pharmacol 1993; 35: 327-30.

40 Jones M, McEwan P, Morgan CL, Peters JR, Goodfellow J, Currie CJ. Evaluation of the pattern of treatment, level of anticoagulation control, and outcome of treatment with warfarin in patients with non-valvar atrial fibrillation: a record linkage study in a large British population. Heart 2005; 91: 472-7.

41 Kubitza D, Becka M, Voith B, Zuehlsdorf M, Wensing G. Safety, pharmacodynamics, and pharmacokinetics of single doses of BAY 59-7939, an oral, direct factor Xa inhibitor. Clin Pharmacol Ther 2005; 78: 412-21. 